\title{
Portable bedside microcomputer system for management of parenteral nutrition in all age groups
}

\author{
P A BALL, D C A CANDY, J W L PUNTIS, AND A S McNEISH
}

Pharmacy Department, Birmingham Children's Hospital and Department of Paediatrics and Child Health, University of Birmingham

SUMMARY A microcomputer program has been designed to provide comprehensive assistance to the clinician in prescribing parenteral nutrition in children of all ages. It is implemented on a fully portable, independent machine that can be taken to the bedside anywhere in the hospital. The user is guided through a standardised prescribing process based on a well tried protocol and taking into account the patient's age, clinical condition, concurrent parenteral infusions, laboratory findings, and enteral intake. A printout is produced for insertion into the patient's clinical record, giving all concerned in the care of the child a clear, legible record of when, how, and by whom the prescription was calculated. The user can review and amend the proposed prescription before printing, giving ultimate control to the prescriber, not the computer.

The system saves time by performing accurately the necessary calculations, and provides financial savings by reducing wastage of parenteral nutrition during the re-introduction of enteral feeding.

The requirements of children needing nutritional support may be met by various combinations of parenteral and enteral nutrition. Many factors affect parenteral nutrition requirements such as the clinical condition and the use of treatments such as phototherapy or infrared heat. Most patients receiving parenteral nutrition also have concurrent intravenous infusions.

The use of computers for parenteral nutrition calculations and record keeping has been widely reported since 1975 . Ward based systems, ${ }^{1-4}$ are concerned with data storage and retrieval, ${ }^{2}$ or guiding the operator through a clinical assessment to arrive at calculated requirements. ${ }^{5-7}$ Pharmacy based systems ${ }^{89}$ calculate the mix of various standard ingredients that will provide the patient's requirements and print all the necessary documentation. The former system obviously requires either a large computer with terminals on every ward ${ }^{35}$ or a dedicated computer system situated on the same ward as the patients receiving parenteral nutrition. ${ }^{5-7}$ It has been our experience that prescribers prefer to perform calculations manually on the ward rather than carry all the necessary information to a remote computer.

At this hospital, the Nutritional Care Team prescribes and supervises parenteral nutrition for eight to 12 patients each day, spread throughout the hospital and ranging from preterm neonates to young adults. All have at least one other medical team involved in their care and this makes good communications vital. No computer facilities exist at ward level. A locally developed, pharmacy based system has been in use since 1981 . $^{10}$ The system, based on an Apple II machine, fitted with two 51/4" floppy disk drives and two printers, is not portable.

We describe a portable microcomputer system designed to provide comprehensive assistance to the prescriber at the bedside.

\section{Materials and methods}

The system selected provides $32 \mathrm{k}$ bytes of user memory on an EPSON HX-20 microcomputer with memory expansion unit, a 20 character $\times 4$ line liquid crystal display, a miniature printer, and a microcassette recorder which is under full software control. The purchase price of the entire system is less than $£ 600$. The program is written in the Microsoft/EPSON version of BASIC supplied with the machine.

The program provides a detailed clinical assessment of the patient, the amount and content of concurrent infusions, and the volume and consti- 
tuents of any enteral intake. It then selects the correct parenteral nutrition requirements from a set of 14 standard tables, ${ }^{1011}$ and offers suggested modifications based on the information provided. A printout (Figure) is then produced for insertion into the patient's clinical record showing the date and time of the assessment, the identity of the prescriber, and the factors taken into account in arriving at the prescription.

Running the program. The system recognises a list of authorised users who each have a LOGIN code. This is not intended to be a security system, it merely allows the operator to be identified on all printouts from the machine.

When a LOGIN code is entered, the machine greets the user and prints a list of patients currently recorded in the machine. The system stores the name, registration number, and full details of the last recorded clinical assessment for up to 10 patients at a time. These details are held overnight on tape in the built in microcassette, so that details of up to 10 patients may be held on each tape, and different users may have their own separate tapes. The patient is selected by entering the number (0-9) or by typing a letter ' $\mathrm{N}$ ' for a new patient.

If a new patient is entered, the system asks for the name, registration number, and weight. The age is then requested from the ranges: less than 1 week; less than 1 month; more than 1 month. If the age is less than one month, the gestation is requested from the ranges: less than 30 weeks; 30 to 40 weeks; more than 40 weeks. If the age is given as less than 1 week, the actual age in days is requested.

The next stage is the clinical assessment, full details of which are recorded within the system. If the operator is satisfied that the condition of an existing patient has not changed, this section may be bypassed. The clinical assessment (detailed below) closely follows the protocol ${ }^{10}{ }^{11}$ which has been in use at this hospital for four years, covering over 6000 patient days of parenteral nutrition. Based upon this
Ivy Feeding

354675

Nutritional Care Tean:

$26 / 11 / 84$

10:07

Seen by Or. D. C. Candy

Prescription allows for:

\section{Uentilation \\ Heart failure \\ Infection}

Concurrent Infusions:

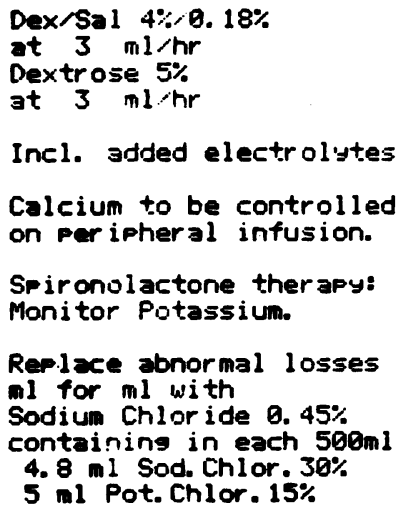

Figure A sample of the computer printout.
Eniteral Feedina:

Feed: Isocal

at $9 \mathrm{ml} / \mathrm{hr}$

Providing:

Protein

$.649 \mathrm{~kg}$

Carbohydrate

$2.59 / \mathrm{kg}$

Fluid

18. $78 \mathrm{ml} / \mathrm{kg}$

Fat

$.83 \mathrm{~g} k \mathrm{k}$

Souium

$.43 \mathrm{mmol} / \mathrm{kg}$

Fotassium

$.64 \mathrm{mmol} / \mathrm{kg}$

Phosphate

$.32 \mathrm{mmol} / \mathrm{k} 3$

Calcium

$.3 \mathrm{mmol} / \mathrm{kg}$

Non N2 Kcal: 18.6

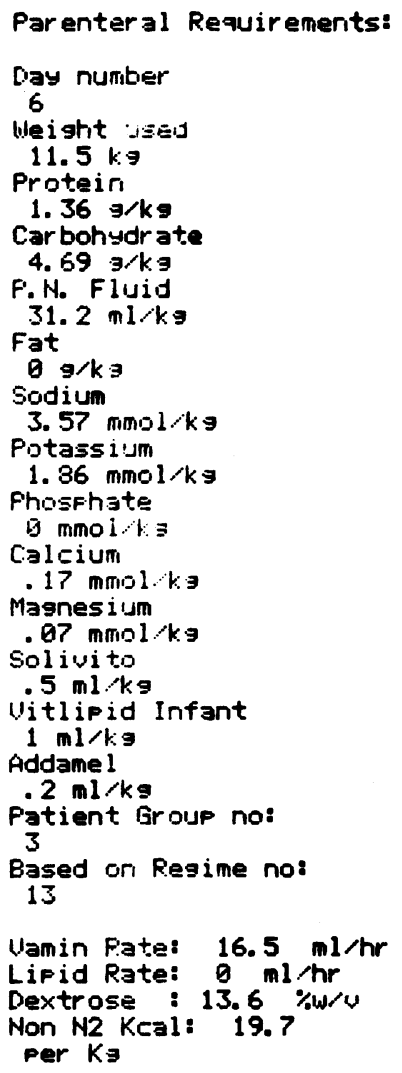

Pharmacy HX-29 Compister 
protocol and the information entered from the keyboard, the computer selects a basic table of requirements, and then offers recommended changes which may be accepted, modified, or ignored by the operator.

The laboratory findings are requested next. These may be entered, estimated, or assumed to be normal.

The number of concurrent infusions excluding abnormal loss replacement is requested, and then for each in turn, the fluid type; the flow rate; and details of added sodium, potassium, or calcium. (The replacement of abnormal loss is calculated by the system on the basis of information supplied in the clinical assessment, and is therefore not included here.)

The type and hourly administration rate of enteral feed is then entered. The machine holds details of commonly used enteral feeds, and has a facility to enter details of any other. Where a non-standard feed is used the dietitians provide a profile of the feed to enter into the computer. The system then asks if any sodium or potassium has been added and calculates the nutrient and electrolyte content of the enteral intake. The operator is given the choice of ignoring or taking into account the enteral intake or volume, or both, when calculating the parenteral requirements.

The machine then offers a series of recommendations based on the clinical assessment and laboratory findings. Some recommendations such as an extra sodium allowance if the patient is receiving frusemide can only be accepted or rejected. Other recommendations can be changed, so that for example the recommended increased fluid allowance for a patient receiving phototherapy is given as $30 \mathrm{ml} / \mathrm{kg}$, but this figure may be changed by the operator.

The operator is then invited to review the final details on the screen before printing. The screen displays full details of the enteral intake, followed by the parenteral intake, giving the operator a final opportunity to modify the prescription. The system prints the details of the prescription and asks 'Change: Yes or No' allowing direct entry of revisions, and giving final control to the operator, not the machine. If no changes are made at this point, the system prints out the consultation report as shown in the Figure. If amendments are made, the system must recalculate the prescription since, for example, increasing the amino acid content would also increase the sodium, potassium, calcium, magnesium, and chloride because the proprietary solution used contains all of these. The operator can again review the details to check the effect of the amendments before proceeding to the printout.

The printout provides a prescription in the correct format (amount of nutrients per $\mathrm{kg}$ ) to be entered into the Apple II pharmacy based system ${ }^{10}$ for calculation and printing.

The system records all the details in its internal files, and moves on to the next patient.

Clinical assessment. The clinical assessment begins with a block of eight yes/no questions:

Is the patient receiving: Ventilation?

Infrared heat?

Phototherapy?

Dialysis?

Is there evidence of: Heart failure?

Renal failure?

Jaundice?

Infection?

The first four questions will later trigger specific recommendations:

Ventilation—reduce daily fluid requirement by $30 \mathrm{ml} / \mathrm{kg}$

Infrared heat-increase daily fluid requirement by $30 \mathrm{ml} / \mathrm{kg}$

Phototherapy-increase daily fluid requirement by $30 \mathrm{ml} / \mathrm{kg}$

Dialysis-consult renal team for fluid allowance.

The latter four cause blocks of additional questions to be asked.

\section{Heart failure}

This question block asks the following questions which are answered by pressing $\mathrm{Y}$ or $\mathrm{N}$.

$\begin{array}{ll}\text { Does the patient have: } & \begin{array}{l}\text { Hepatomegaly? } \\ \text { Tachycardia? } \\ \text { Tachypnoea? } \\ \text { Triple rhythm? }\end{array} \\ \text { Is the patient receiving: } & \begin{array}{l}\text { PDA murmur? } \\ \text { Frusemide? }\end{array} \\ \text { Spironolactone? }\end{array}$

A positive answer to two or more of these questions causes the 'heart failure' recommendations to be activated. This recommends fluid intake to be reduced by $25 \%$. In addition, if the patient is receiving frusemide, an increase in the sodium intake of $1 \mathrm{mmol} / \mathrm{kg} /$ day is recommended. In the presence of spironolactone treatment, the operator is warned to monitor potassium closely.

\section{Jaundice}

The section asks two questions:

Is the serum bilirubin $1 .<100 \mu \mathrm{mol} / 1$ ?

2. $>100 \mu \mathrm{mol} / \mathrm{l}$ ?

3. $>170 \mu \mathrm{mol} / \mathrm{l}$ ?

Is there clinical evidence of liver failure?

If clinical liver failure is proved or suspected, the system suggests consultant referral and, in the meantime, a reduction in amino acid intake to $0.25 \mathrm{~g} / \mathrm{kg} /$ day and fat intake of $0.5 \mathrm{~g} / \mathrm{kg} /$ day with 
sufficient carbohydrate to maintain serum glucose above $10 \mathrm{mmol} / \mathrm{l}$.

If the serum bilirubin concentration is between 100 and $170 \mu \mathrm{mol} / \mathrm{l}$ the intravenous fat intake is reduced to $0.5 \mathrm{~g} / \mathrm{kg} /$ day. If the value is greater than $170 \mu \mathrm{mol} / \mathrm{l}$, the fat intake is stopped completely, and topical sunflower oil should be applied to all available skin twice daily to prevent essential fatty acid deficiency.

\section{Infection}

These questions are asked:

Does the patient have: Pyrexia?

Acidosis?

Neutrophilia?

Low skin temperature?

Blood glucose unstable?

If the answer to two or more of these questions is positive, the recommendation is triggered and the system asks:

Is the infection controlled or uncontrolled?

If the infection is considered to be uncontrolled, the operator is advised to check that the other medical teams involved have noted the infection, and that the normal investigations have been ordered. Intravenous amino acids and fat should be stopped, and the fluid increased by $10 \mathrm{ml} / \mathrm{kg} /{ }^{\circ} \mathrm{C}$ of pyrexia. Once the infection is under control, amino acids and fat are restarted, but the fat is limited to $0.5 \mathrm{~g} / \mathrm{kg} /$ day (sufficient to prevent essential fatty acid deficiency) until the infection is resolved.

\section{Renal failure or peritoneal dialysis}

The system recommends that the renal team be consulted for the allowable fluid and electrolyte intake. The parenteral nutrition should be based on $0.5 \mathrm{~g} / \mathrm{kg} /$ day of amino acid with no added sodium, potassium, or trace elements.

In each case, rejection of the recommendation causes the operator to be asked for an alternative figure, which may also be entered as zero, effectively rejecting the recommendation.

Laboratory assessment. Based upon the serum sodium, potassium calcium, albumin, and glucose, and the urine sodium and potassium concentrations, the system offers specific recommendations.

If, for example, the plasma sodium value is high, the system asks if the patient is dehydrated, and if so to increase the fluid until corrected. If the sodium is low, the operator is asked if the patient is in fluid overload. If yes, fluid is decreased until corrected. In addition, if the sodium is below $133 \mathrm{mmol} / \mathrm{l}$, in the absence of fluid overload, the system calculates a supplement to return it to the normal range.
If the serum potassium concentration is greater than $6 \mathrm{mmol} / \mathrm{l}$, the operator is asked if this has been confirmed on a sample of venous blood, since heelprick capillary samples are normally used. If not, the option is provided to continue, assuming this to be an incorrect result, or to abort the calculation, and await an urgent repeat investigation. If the potassium value is less than $3 \mathrm{mmol} / \mathrm{i}$, a supplement of $1 \mathrm{mmol} / \mathrm{kg} / \mathrm{day}$ is recommended.

If the urine potassium concentration is greater than the urine sodium, the system recommends that the sodium be supplemented by $1 \mathrm{mmol} / \mathrm{kg} / \mathrm{day}$ until the ratio is corrected.

The system then performs a check to ensure that the calculated requirements can be prepared within the available fluid allowance. If this is not possible, the nutritional content is reduced in balanced proportions to the maximum that can be accommodated in the allowable fluid.

\section{Discussion}

We have described a portable microcomputer system designed to guide an experienced prescriber through a standardised parenteral nutrition prescribing process, which ensures that nothing is overlooked and performs the required calculations correctly and accurately en route. All the advice offered by the system can be ignored, and ultimate control of the prescription rests with the operator, not with the computer.

Concurrent infusions can be a serious problem if not accurately accounted. They may range from a slow flush of five per cent dextrose to maintain the patency of a monitoring line, to a secondary infusion route for parenteral medication. Although it may be quite safe to ignore such small volumes in larger children and adults, in a preterm neonate, a 1 $\mathrm{ml} /$ hour flush may constitute as much as half of the child's fluid requirement for 24 hours. Using this system, the operator can be sure of the total amounts of fluid, sodium, potassium, and dextrose received by the patient in a given period.

The process of re-introducing enteral feeding can take weeks and occasionally months. In the past the practice has been to exchange parenteral feed $\mathrm{ml}$ for $\mathrm{ml}$ with enteral feed. As the enteral feed increases, so the parenteral feed is reduced in total. The enteral feed composition may differ quite considerably, however, from the parenteral. The system will automatically adjust the parenteral feed to reflect any enteral intake and the operator is fully aware of what the patient will receive in total. Before the introduction of this system, for simplicity during the changeover, a parenteral nutrition solution would be ordered containing full 24 hour requirements, 
and the unused proportion would be wasted. Use of this system can largely eliminate this wastage. If the unit achieved a one per cent saving in ingredient costs at this hospital it would pay for itself within one year. In practice, much greater savings should be possible.

The insertion of the printout in the patient's clinical record communicates clearly and legibly to all medical teams concerned the basis on which the parenteral nutrition solution has been calculated, by whom, and what effect alteration to flow rates on concurrent infusions or changes of solution used are likely to have.

The system was designed to be complementary to the existing Apple II system. ${ }^{10}$ On its own, the system described in this paper can also provide a calculated printout of ingredients to assemble. The additional features provided by the Apple II system make its continued use valuable to a large unit such as Birmingham Children's Hospital, but in a smaller unit the HX-20 system could readily operate alone.

An editor program has been written to allow changes to be made to the operator list, the stored enteral feed data etc without programming ability. This can also be used to change the wording of many of the questions asked by the system.

The system is based on a well tried protocol ${ }^{11}$ with over 6000 patient days of clinical experience to date. It is very simple to change the protocol if desired using the editor program, provided that the desired protocol can be expressed in the same format. This also prevents errors being introduced by attempting to alter program lines directly.

Flexibility. MacMahon ${ }^{7}$ states that programs written in any version of BASIC can readily be transferred to other machines by those with a rudimentary knowledge of programming. This is perhaps an overstatement of the position. Many of the more recent versions of BASIC have features not possessed by the earlier implementations, and the screen and file handling features of BASIC tend to be machine specific.

To assist in flexibility, the program is written using features common to most dialects of BASIC, avoiding the use of some features which, though possessed by the EPSON and many more modern machines, are not available on, for example, the Sinclair SPECTRUM as used by MacMahon, or the very popular Apple II series of computers (unless using the $\mathrm{CP} / \mathrm{M}$ operating system and Microsoft Corporation's M-BASIC). The screen handling has been largely confined to clearing the screen, and printing one question or statement on it. This is essential when dealing with a screen of only 20 characters $\times 4$ lines, but does also facilitate flexibility.

In addition, the program listing will only be supplied with detailed notes explaining what each section does, making transfer to other machines as straightforward as possible. Full details of program availability are given below.

The microcomputer for parenteral nutrition seems to have found a home in the neonatal intensive care unit. ${ }^{2-6}$ Use of the EPSON HX-20 allows the microcomputer to leave the neonatal unit and extend computer facilities to the bedside of all children requiring nutritional care.

We thank Dr Roger Flinn for seeding the original idea, and introducing the team to the HX-20 machine.

The program will be supplied as either a listing with explanatory notes $(£ 25.00)$ or cassette (for HX-20), listing, explanatory notes, and system handbook $(£ 50 \cdot 00)$. All proceeds benefit the Birmingham Children's Hospital pharmacy research fund.

\section{References}

1 Danford DE. Computer applications to medical nutrition problems. JPEN 1981;5:441-6.

2 Janik DS, Swarner OW, Henriksen BS, Wymam ML. Computerised newborn intensive care data recording and reporting. II: An online system. J Pediatr 1979;94:328-30.

${ }^{3}$ Sharp DS, German JC. Computer utilization for intravenous nutrition in surgical neonates: Preliminary report. J Pediatr Surg 1977;12:189-93.

4 Wilson FE, Yu VY, Hagwood S, Adamson TM, Wilkinson MH. Computerised nutritional data management in neonatal intensive care. Arch Dis Child 1983;58:732-6.

5 Gale R, Gale J, Branski D, Armon Y, Zellingher J, Roll D. An interactive microcomputer program for calculation of combined parenteral and enteral nutrition for neonates. J Pediatr Gastroenterol Nutr 1983;2:653-8.

' Giacoia GP, Chopra R. The use of a computer in parenteral alimentation of low birth weight infants. JPEN 1981;5:328-31.

MacMahon P. Prescribing and formulating neonatal intravenous feeding solutions by microcomputer. Arch Dis Child 1984; 59:548-52.

${ }^{\times}$Rich DS, Karnack CM, Jeffrey LP. An evaluation of a microcomputer in reducing the preparation time of parenteral nutrition solutions. JPEN 1982;6:71-5.

9 Brouwer D. Neonatal total parenteral nutrition: a program for computer generated profiles. Hospital Pharmacy 1984;19:271-2.

11 Ball PA, De Silva DG, Candy DCA, McNeish AS. The microcomputer: an aid to pacdiatric parenteral nutrition. International Journal of Clinical Monitoring and Computing 1985 ; in press.

"Hughes CA. Parenteral nutrition. In: Insley J, Wood B, eds. A paediatric vade mecum. London: Lloyd-Luke, 1982:60-7.

Correspondence to Professor A S McNeish, Institute of Child Health, Francis Road, Edgbaston, Birmingham, B16 8ET.

Received 14 December 1984 